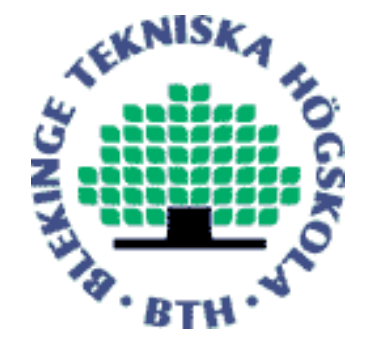

Copyright (C) 2010 IEEE.

Citation for the published paper:

The empowered user - The critical interface to critical infrastructures

Rune Gustavsson, Björn Ståhl

The Fifth International CRIS conference on Critical Infrastructures - Interacting Critical Infrastructures for the 21st Century.

2010 Beijing

This material is posted here with permission of the IEEE. Such permission of the IEEE does not in any way imply IEEE endorsement of any of BTH's products or services Internal or personal use of this material is permitted. However, permission to reprint/republish this material for advertising or promotional purposes or for creating new collective works for resale or redistribution must be obtained from the IEEE by sending a blank email message to pubs-permissions@iee.org.

By choosing to view this document, you agree to all provisions of the copyright laws protecting it. 


\section{The empowered user - The critical interface to critical infrastructures}

\author{
Rune Gustavsson \\ Blekinge Institute of Technology \\ Ronneby, Sweden \\ Email: rune.gustavsson@bth.se \\ Royal Institute of Technology \\ Stockholm, Sweden \\ Email: rune.gustavsson@ics.kth.se
}

\author{
Björn Ståhl \\ Blekinge Institute of Technology \\ Ronneby, Sweden \\ Email: bjorn.stahl@bth.se
}

\begin{abstract}
Future smart grids will enable increased energy efficiency due to utilization of vast amounts of Distributed Energy Sources (DER) and Renewable Energy Sources (RES) partially controlled by empowered customers. Smart generation and distribution systems will connect energy providers with customers and other stakeholders utilizing new kinds of smart monitoring, coordination and data management infrastructures in service clouds. The service clouds are based on suitable virtualization techniques that could to used to harness critical interdependencies between critical infrastructures by introducing smart programmable interfaces. Smart meters enable empowerment of end users. Smart meters are, however, also access points to the service cloud. The paper addresses some security challenges of energy service clouds and smart meters.

The layout of the paper is as follows; Setting the Scene briefly introduces the notion of Smart grids in relation to currently ongoing international efforts. In the section on Customer empowerment and Smart meters we elaborate on emergent security concerns related to smart meters. The next section on Smart Grids as Service Clouds introduces service clouds as a viable setting of smart grids. The section on Experimental environments introduces two experimental platforms addressing complementary views on Smart grids - customer empowerment and management of vast amounts of RES. The paper ends with some concluding remarks.
\end{abstract}

This paper describes work in progress.

\section{Setting The SCEne}

The following three figures illustrate some challenges related to identifying interfaces between critical infrastructures. The figures show different views on future Smart grids. Fig. 1 is adopted from deliverables from the EU funded TN SEESGEN-ICT (Supporting Energy Efficiency in Smart Generation grids through ICT) illustrates the main characteristics behind the transition of electric grids from today to tomorrow.

In Fig. 1 the main stakeholders and roles are depicted as well as the path of transformation from Today to Tomorrow related to effects due to the unbundling of the energy market. The deregulations and increased intelligence in the Transmission and Distribution networks enabled by smart programmable electronic components and smart ICT information management systems are the two main drivers of this transition. Fig. 1 depicts the main architectural components related to the energy flow of the future Smart Grid. The following Fig. 2 outlines the information flows between groups of stakeholders to enable and support new business models as well as empowerment of the customers.

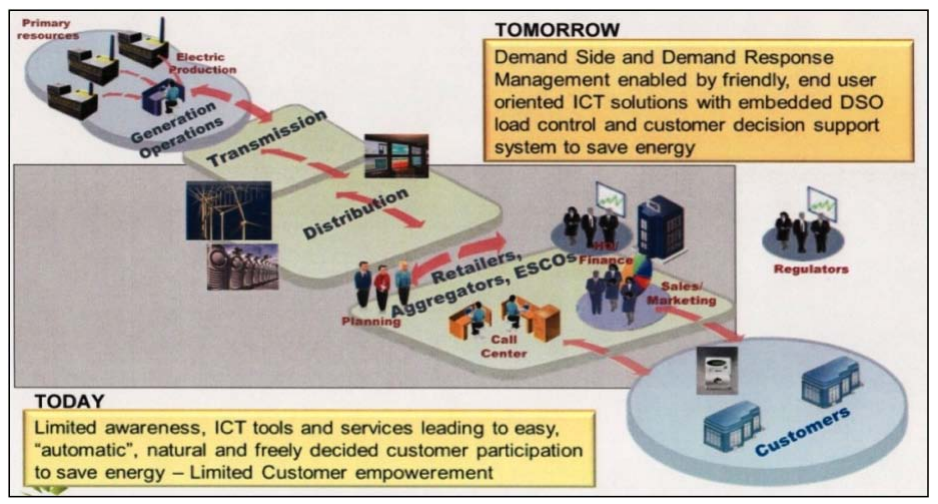

Fig. 1. Main drivers behind the transition towards Smart Grids

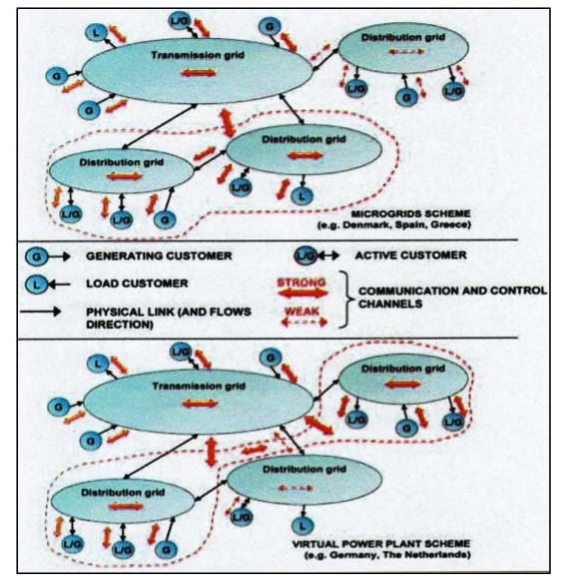

Fig. 2. Information flows in future Smart Grids (adapted from SEESGENICT[1] deliverables)

From Fig. 3 it follows that Smart grid data availability places considerably more stringent demands on the communi- 


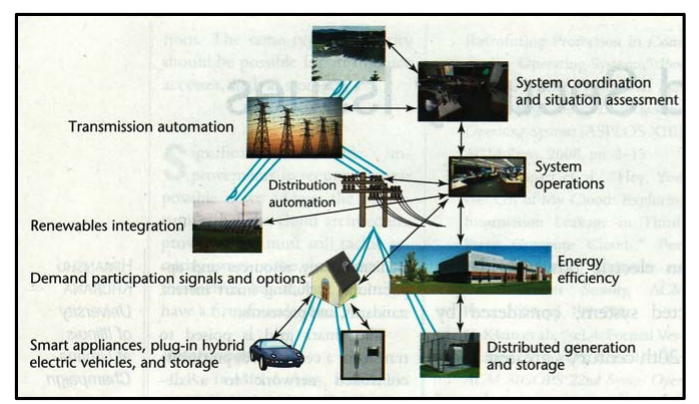

Fig. 3. The physical layers and communication / control system of the Smart grid

cation and control systems than traditional supervisory control and data acquisition (SCADA) systems. From Fig. 1 and Fig. 2 respectively, we firstly deduce that new stakeholders, e.g., Aggregators and Retailers, will interact in Smart Grids between e.g., DSOs and Customers, and secondly, we will have flexible configuration of stakeholders, such as Virtual Power Plants (VPP). Finally, we will need communication networks that must support the energy flow (SCADA) as well as the customer-based business information flows. In short, present SCADA systems have to be supported by novel ICT based information systems to meet the requirements of future Smart Grids for empowerment of customers. As a consequence, the monitoring task of present day energy systems has to be reassessed and re-designed. To that end we propose to extend the monitoring task by introducing the concept and mechanism of Service Level Agreements (SLAs) to:

- Allow flexible grouping of stakeholders

- Allow flexible empowerment of users

From Fig. 1 - 3 above it follows that the critical infrastructures involved in future Smart grids (energy systems, control systems, information processing systems and business systems) are typically layered open and loosely coupled systems implying that we have a recursive set of interfaces between components allowing for hard to predict control feedback loops. The mechanisms of programmable interfaces and virtualization are identified candidates to design and implement the relevant service clouds of future Smart grids.

\section{Customer EMPowerment AND SMART METERS}

The Smart grid is poised to transform a centralized, producer-controlled net-work to a decentralized consumerinteractive network that is supported by a fine-grained monitoring (Fig. 1). The Phasor Measurement Units (PMUs) in the power transmission grid and the Advanced Metering Infrastructure (AMI) in the power distribution system will provide the smart power grid an "Magnetic Resonance Imaging" of the grid compared to the "x-ray" available from SCADA technology. In short we will have methods and tools far more advanced than those of today to monitor and reason about the state of a Smart grid. However, in addition, smart-building and home automation technology extends control and monitoring to the end-user level. Thus widely used digital control and communication technologies provide operators unprecedentedly abundant information and inform them of the status of the multitude of devices connected to the power grid, such as generators, breakers and home appliances. In short, AMI technologies can empower the end user in taking measures to increase energy efficiency and save costs as the AMI provides a customer-oriented local smart SCADA system. On the other hand AMI technologies are potential threats to Smart grids as they can exploit vulnerabilities of the service cloud.

\section{A. Smart meter vulnerabilities}

The vulnerabilities of Smart meters have been described in several reports, e.g. a white paper from Inguardians[2]. There is also a growing concern regarding exploiting in-home data generated by, e.g., Smart grids[3].

\section{B. Identified Barriers}

Several international assessments of the transition from present day energy systems towards future Smart Grids have identified a set of barriers that have to be resolved, for instance[8]:

- Regulatory barriers. New types of stake holders and new kinds of business processes[1]

- Technical barriers. Architectures and technologies supporting new kinds of ICT systems complementing and enforcing SCADA systems[4]

- Customer acceptance. Trust in value-added services provided[3]

- Lack of experiences. Today, there is a lack of experience from large-scale field tests or demonstrators addressing key challenges on the road towards smart grids. For instance, the roles and amounts of DER or RES that can be utilized and trustworthy managed.[6]

In this paper we describe a configurable Agent-based platform addressing coordination in future smart grids in the form of monitoring SLAs. The initial focus is on customer empowerment and acceptance and to gain experiences of possible new business processes in Smart Grids

\section{SMART GRIDS AS SERVICE ClOUdS}

There are several ongoing international $R \& D$ efforts on cloud computing. In principle we have three kinds of clouds:

- Software as a Service (SaaS)

- Platform as a Service (PaaS)

- Infrastructure as a Service (IaaS)

Cloud computing has particular characteristics that distinguish it from classical resource and service provisioning environments :

- it is (more-or-less) infinitely scalable;

- it provides one or more of an infrastructure for platforms, a platform for applications or applications (via services) themselves;

- thus clouds can be used for every purpose from disaster recovery/business continuity through to a fully outsourced ICT service for an organization; 
- clouds shift the costs for a business opportunity from CAPEX to OPEX which allows finer control of expenditure and avoids costly asset acquisition and maintenance reducing the entry threshold barrier;

- currently the major cloud providers had already invested in large scale infrastructure and now offer a cloud service to exploit it;

- as a consequence the cloud offerings are heterogeneous and without agreed interfaces;

- cloud providers essentially provide datacentres for outsourcing;

- there are concerns over security if a business places its valuable knowledge, information and data on an external service;

- there are concerns over availability and business continuity - with some recent examples of failures;

- there are concerns over data shipping over anticipated broadband speeds.

A basic technology in designing and implementing clouds is virtualization as a technology to provide better resource utilization and more flexible services. Due to virtualization potentially competing stake holders can safely manage the topology of their virtual networks while sharing physical infrastructures (IaaS) provided by a system owner. While these virtualized environments offer many benefits, they also represent very complex and dynamic systems, and so pose significant management and control challenges.

A concrete example of cloud computing is Amazon Elastic Computer Cloud (Amazon EC2)[5] From Fig. $1-3$ and the discussions above we suggest that a viable design and implementation strategy for Smart grids could be development of an Infrastructure as a Service (Iaas).

A recent overview of Cloud computing is given in[9]. The paper list some pros and cons of cloud computing of interest to Smart grids. The paper also give a list of Top 10 ostacles and opportunities for growth of cloud computing.

\section{EXPERIMENTAL ENVIRONMENTS}

From figures $1-3$ and the discussion in section 1, we can identify different views of future Smart grids. From one point of view we have assembles of smart equipment that has to be coordinated and managed to provide energy-based services to the customer. From another point of view we have advanced ICT systems supporting business processes and customer empowerment. At the same time we have to support different sets of views from the stakeholders of future smart grids. In short, smart grids are examples of future Software - Intensive Systems, an identified Research area of the Future and Emerging Technologies (FET) Unit of the European Commission (http://interlink.ics.forth.gr)[11]. In our introduction we introduced the concept of Service Level Agreements (SLAs) as a mechanisms of high-level coordination between groups of stakeholders and/or monitoring of regulatory policies. We implement our SLA-Agents platform using the JACK industry standard. Of specific interest to us is the identification of SLAs and policies aiming at empowerment of the customer but also protecting information integrity and availability. Obviously, there are interconnections between empowerment of the user and technical constraints of the grid. As an example: An empowerment service to the customer could be to change the amount of RES-generated energy to be used ("green energy"). However, the allowed change of RES must be subjected to proper voltage control. In short, the allowed services to customers have to be balanced by services provided by, e.g., an aggregator. That is, we will have crucial interdependencies between SLAs involving different stakeholders. The views and experiments we can conduct using the SLA-Agents platform has thus to be complemented by experiments, and findings, focusing in the technical parts of the smart grid. To that end we use another platform EXP-II described in a companion paper Experimenting with Infrastructures in this Proceeding. The EXP-II set up will allow, among other things, to set up and perform different types of security experiments related to AMIs.

\section{CONCLUSIONS AND FURTHER WORK}

Future Smart grids pose several challenges to be addressed and harnessed. Furthermore several barriers of uptake and acceptance of Smart grids have been identified. In fact, a Smart grid can be seen as a combination of several interacting critical infrastructures. Obviously, identification and proper design of critical interfaces between those infrastructures have a key role in ensuring resilient Smart grids.

We argue that Smart Metering Infrastructures is indeed a crucial interface to address both to ensure user empowerment and hence acceptance but also to address crucial security vulnerabilities of Smart grids. Furthermore, we also argue that cloud computing (Infrastructure as a Service) might be a cost efficient way to design, implement and run future smart grids. To enable relevant experiments we are at present implementing two platforms SLA-Agents and EXP-II.

\section{REFERENCES}

[1] [Online]. Available: http://seesgen-ict.erse-web.it/

[2] [Online]. Available: http://inguardians.com/pubs/AMI_Attack_Methodology.pdf

[3] [Online]. Available: http://www.smartgrids.eu

[4] [Online]. Available: http://www.integral-eu.com

[5] "Amazon web services home page." [Online]. Available: http://aws.amazon.com

[6] "The european future internet initiative (efi)."

[7] "The future of cloud computing. opportunities for european cloud computing beyond 2010," EC Expert Group Report, December 2009. [Online]. Available: http://cordis.europa.eu/fp7/ict/ssai/docs/cloudreport-final.pdf

[8] "Technology action plan: Smart grids," Report to the Major Economies Form (MEF) on Energy and Climate by Italy and Korea, December 2009.

[9] M. Armbrust, A. Fox, R. Griffith, A. D. Joseph, R. Katz, A. Konwinski, G. Lee, D. Patterson, A. Rabkin, I. Stoica, and M. Zaharia, "A view of cloud computing," Commun. ACM, vol. 53, no. 4, pp. 50-58, 2010.

[10] M. A. Lisovich, D. K. Mulligan, and S. B. Wicker, "Inferring personal information from demand-response systems," IEEE Security and Privacy, vol. 8, pp. 11-20, 2010.

[11] M. Wirsing, J.-P. Banâtre, M. M. Hölzl, and A. Rauschmayer, Eds., Software-Intensive Systems and New Computing Paradigms - Challenges and Visions, ser. Lecture Notes in Computer Science. Springer, 2008, vol. 5380 